\title{
Reverse ground-state excited-state emission transition dynamics in two-section quantum dot semiconductor lasers: simultaneous two- state mode-locking and state-switching via a resistor Self-Electro- optic Effect Device (SEED)
}

\author{
Stefan Breuer, Member, IEEE, Mattia Rossetti*, Wolfgang Elsässer, Senior Member, IEEE, Lukas \\ Drzewietzki, Paolo Bardella*, Ivo Montrosset*, Member, IEEE, and Mark Hopkinson**, Member, IEEE \\ Technische Universität Darmstadt, Schlossgartenstraße 7, 64289 Darmstadt, Germany, \\ Phone ++49(0)6151-16-4086, FAX ++49(0)6151-16-3022, E-Mail: stefan.breuer@physik.tu-darmstadt.de \\ *Politecnico di Torino, Corso Duca degli Abruzzi 24, 10129 Torino, Italy \\ **University of Sheffield, Mappin Street, Sheffield, S1 3JD, United Kingdom
}

\begin{abstract}
Reverse emission state transition and simultaneous two-state mode-locking in a twosection quantum dot semiconductor laser is demonstrated. By exploiting a resistor Self-Electrooptic Effect Device (SEED) configuration, novel state-switched mode-locking and full emission state control is realized.
\end{abstract}

Keywords: two-state passive mode-locking, dual-state emission, optical switching, quantum dot emission hierarchy, two-section quantum dot semiconductor laser, picosecond pulse generation.

Semiconductor lasers based on quantum dots (QD) exhibit a particular interesting dynamical behavior due to the existence of ground-state (GS) and excited-state (ES) in the dot energy scheme [1,2]. The coexistence of GS and ES mode-locking (ML) has been reported where a transition from GS to ES was found by increasing the gain current of a two-section QD semiconductor laser [3,4]. In this contribution, reverse emission state transition of a 3.3 $\mathrm{mm}$ long two-section InAs/InGaAs QD laser with a $0.3 \mathrm{~mm}$ long saturable absorber (SA) section is presented. ML starts first on the energetically higher first ES. With increasing gain current additionally the energetically lower GS starts ML. This particular reverse GS/ES emission state dynamics is observed at a SA bias of zero Volt (short circuit). The corresponding optical spectra evolution is depicted in Fig. 1, indicating a spectral separation of the

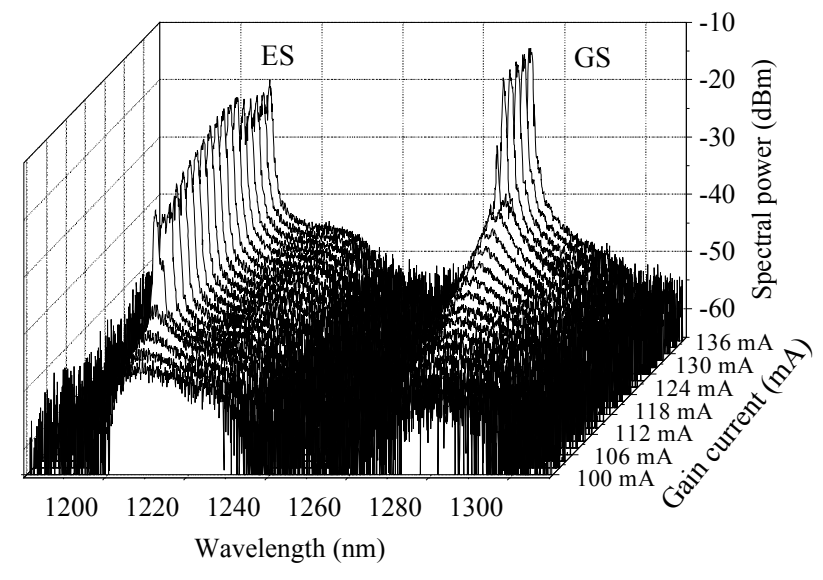

Fig. 1. Experimentally measured optical spectra evolution with increasing gain current and at an absorber voltage of zero Volt.

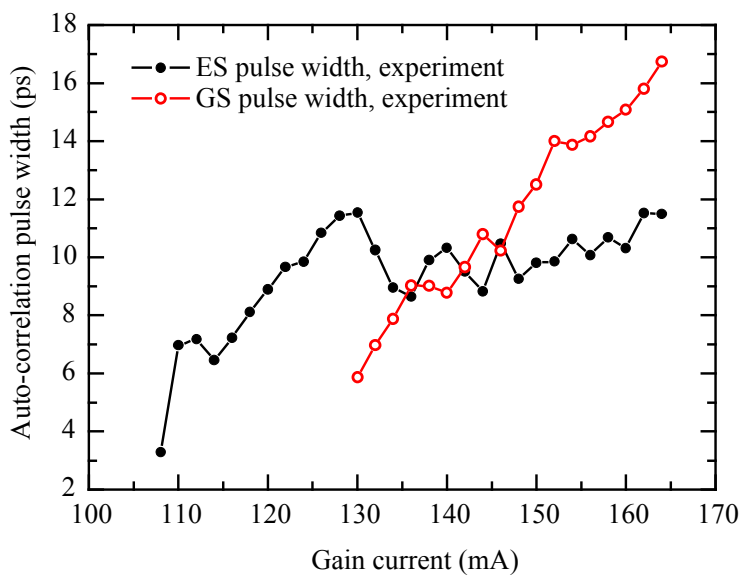

Fig. 2. Experimentally measured spectrally-resolved pulse widths obtained at an absorber voltage of zero Volt.

quantized states of $60 \mathrm{~nm}$. Experimentally observed state-resolved picosecond pulse widths in mode-locked operation are depicted in Fig. 2. At the onset of two-state ML, appreciably shorter pulse widths are found for the GS as compared to the ES at the same gain current. Additionally, a considerable shortening of the ES pulse widths is observed when GS ML starts. A huge coexistence regime and temporal simultaneity of mode-locked picosecond pulses on both GS and ES is experimentally verified. This reverse emission state transition scenario is attributed to the particularly technologically designed losses of the laser structure as well as to the low saturated GS gain induced 
by a significant wavelength chirp in the multiple QD layers. These experimentally observed, particularly interesting state-resolved reverse emission state transition dynamics as well as the picosecond pulse properties are confirmed by time-domain travelling-wave equation modeling [5-7]. A photon-pumping process in the SA section is identified which stimulates GS emission while ES emission takes place: ES photons are generated in the gain section that can be absorbed in the SA section. The ES photo-generated carriers can then either be excited to higher energy states or relax into the GS while reducing GS absorption [8]. This identified physical mechanism that is responsible for the novel reverse state transition has a particularly fascinating consequence: the realization of complete emission state control of the specifically designed multi-layer two-section QD laser by electrically shortening the SA section via an external variable resistor, forming a resistor SEED configuration $[9,10]$. The simplified experimental set-up is depicted in Fig. 3. By variation of the external resistor value both ES and GS carrier dynamics can be appreciably controlled. The experimentally realized optical spectra evolution is depicted in Fig. 4. At low external resistance

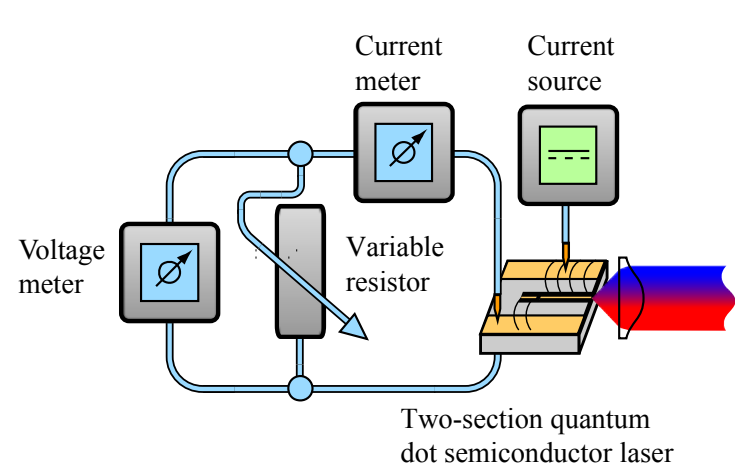

Fig. 3. Experimental set-up for state-switched ML by exploiting a resistor SEED configuration on a two-section QD laser. A variable external resistor is placed across the SA section.

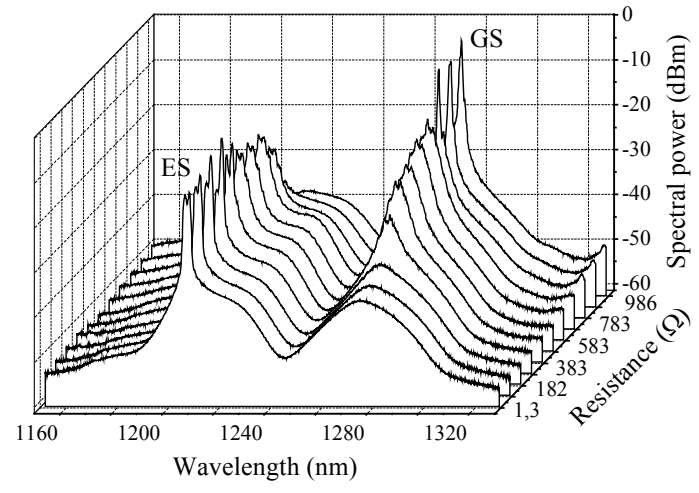

Fig. 4. Experimentally measured optical spectra evolution with increasing external resistor value at a gain current of $110 \mathrm{~mA}$.

values, ES ML is observed. By increasing the external resistance value the photon-pumping process starts to reduce GS absorption in the SA section. At $580 \mathrm{Ohm}$ both ES and GS show lasing. By further increasing the external resistor value up to $986 \mathrm{Ohm}$ only GS lasing and $\mathrm{ML}$ is obtained. By exploiting this novel resistor SEED configuration in a two-section QD laser, state-switched ML is demonstrated.

\section{ACKNOWLEDGEMENTS}

This work was supported by the EU Sixth Framework Program project "NANO UB-SOURCES" (Grant No. 017128), as well as by the EU Seventh Framework Program "FAST-DOT" (Grant No. 224338). Processing of the two-section QD structures was carried out by M. Krakowski from Alcatel Thales III-V lab. Fruitful discussions with M.A. Cataluna are gratefully acknowledged.

\section{REFERENCES}

[1] Bimberg, D., "Semiconductor quantum dots”, Ch. 2 in 'Optical Fiber Telecommunications V A: Components and Subsystems' (I. Kaminov, T. Li, A. Willner, Eds.), pp. 23 (2008).

[2] Rafailov, E. U., Cataluna, M. A., Sibbett, W., "Mode-locked quantum dot lasers," Nature Photon. 1, 395-401 (2007).

[3] Cataluna, M. A., McRobbie, A. D., Sibbett, W., Livshits, D. A., Kovsh, A. R., Rafailov, E. U., "New mode locking regime in a quantum-dot laser: enhancement by simultaneous cw excited-state emission," CLEO/QELS 2006, Long Beach, Calif., 1-2 (2006).

[4] Cataluna, M. A., Nikitchev, D. I., Krestnikov, I., Livshits, D. A., Kovsh, A. R., Rafailov, E. U., "Dual-wavelength mode-locked GaAsbased quantum-dot laser," CLEO Europe - EQEC 2009, Munich, CB4 (2009).

[5] Rossetti, M., Bardella, P., Montrosset, I., "A time-domain travelling-wave model including multi-population rate-equations for passively mode-locked quantum-dot lasers," 1st EOS Topical Meeting on Lasers, Capri, Italy (2009).

[6] Rossetti, M., Bardella, P., Gioannini, M., Montrosset, I., "Time Domain Travelling Wave Model for Simulation of Passive Mode Locking in Quantum Dot Lasers," CLEO Europe - EQEC 2009, Munich, CF.P.21 (2009).

[7] Breuer, S., Rossetti, M., Elsaesser,W., Drzewietzki, L., Bardella, P., Montrosset, I., Krakowski, M., Hopkinson, M., "Reverse ground-state excited-state transition dynamics in two-section quantum dot semiconductor lasers: mode-locking and state-switching", SPIE Photonics Europe 2010, Brussels, 7720-35 (2010).

[8] Breuer, S., Rossetti, M., Elsaesser,W., Drzewietzki, L., Bardella, P., Montrosset, I., Krakowski, M., Hopkinson, M., "Reverse ground-state excited-state transition dynamics in mode-locked two-section quantum dot semiconductor lasers," Opt. Lett., submitted (2010).

[9] Miller, D. A. B., "Quantum-well self-electro-optic effect devices”, Opt. and Quant. Electronics 22, S61-S98 (1990).

[10] Breuer, S., Elsaesser, W., Hopkinson, M., "State-Switched Mode Locking of a Two-Segment Quantum Dot Laser via a Self-Electro-Optical Quantum Dot Absorber," Electron. Lett. 46 (2), 161-162 (2010). 